\title{
Article \\ A Class of Quasilinear Equations with Riemann-Liouville Derivatives and Bounded Operators
}

\author{
Vladimir E. Fedorov ${ }^{1,2, *\left(\mathbb{D}, \text { Mikhail M. Turov }^{1} \mathbb{D} \text { and Bui Trong Kien }\right.}{ }^{3}$
}

1 Department of Mathematical Analysis, Mathematics Faculty, Chelyabinsk State University, Kashirin Brothers St., 129, 454001 Chelyabinsk, Russia; turov_m_m@mail.ru

2 Laboratory of Functional Materials, South Ural State University (National Research University), Lenin Av., 76, 454080 Chelyabinsk, Russia

3 Department of Optimization and Control Theory, Institute of Mathematics of the Vietnam Academy of Sciences and Technologies, 8 Hoang Quoc Viet Road, Caugiay District, Hanoi 10307, Vietnam; btkien@math.ac.vn

* Correspondence: kar@csu.ru

Citation: Fedorov, V.E.; Turov, M.M.; Kien, B.T. A Class of Quasilinear Equations with Riemann-Liouville Derivatives and Bounded Operators. Axioms 2022, 11, 96. https://doi.org/ 10.3390/axioms11030096

Academic Editor: Chris Goodrich

Received: 31 January 2022

Accepted: 23 February 2022

Published: 24 February 2022

Publisher's Note: MDPI stays neutral with regard to jurisdictional claims in published maps and institutional affiliations.

Copyright: () 2022 by the authors. Licensee MDPI, Basel, Switzerland. This article is an open access article distributed under the terms and conditions of the Creative Commons Attribution (CC BY) license (https:// creativecommons.org/licenses/by/ $4.0 /)$.

\begin{abstract}
The existence and uniqueness of a local solution is proved for the incomplete Cauchy type problem to multi-term quasilinear fractional differential equations in Banach spaces with RiemannLiouville derivatives and bounded operators at them. Nonlinearity in the equation is assumed to be Lipschitz continuous and dependent on lower order fractional derivatives, which orders have the same fractional part as the order of the highest fractional derivative. The obtained abstract result is applied to study a class of initial-boundary value problems to time-fractional order equations with polynomials of an elliptic self-adjoint differential operator with respect to spatial variables as linear operators at the time-fractional derivatives. The nonlinear operator in the considered partial differential equations is assumed to be smooth with respect to phase variables.
\end{abstract}

Keywords: multi-term fractional differential equation; quasilinear equation; Riemann-Liouville fractional derivative; defect of Cauchy type problem; fixed point theorem; initial-boundary value problem

\section{Introduction}

In recent decades, problems with fractional derivatives have been studied by many authors [1-5]. Now fractional integro-differential calculus is an important tool in modeling various phenomena that arise in physics, chemistry, mathematical biology, engineering, etc. (see e.g., [6,7]).

The purpose of this paper is to study the local unique solvability of initial value problems for multi-term equations in Banach spaces with fractional Riemann-Liouville derivatives $D_{t}^{\beta} z, \beta>0$, fractional Riemann-Liouville integrals $J_{t}^{\beta} z, \beta \geq 0$, and with nonlinearity, which depends on fractional derivatives of lower orders

$$
\begin{aligned}
D_{t}^{\alpha} z(t) & =\sum_{j=1}^{m-1} A_{j} D_{t}^{\alpha-m+j} z(t)+\sum_{l=1}^{n} B_{l} D_{t}^{\alpha_{l}} z(t)+\sum_{s=1}^{r} C_{s} J_{t}^{\beta_{s}} z(t) \\
& +F\left(t, D_{t}^{\alpha-m} z(t), D_{t}^{\alpha-m+1} z(t), \ldots, D_{t}^{\alpha-1} z(t)\right) .
\end{aligned}
$$

Operators $A_{j}, j=1,2, \ldots, m-1, B_{l}, l=1,2, \ldots, n, C_{s}, s=1,2, \ldots, r$ are supposed to be bounded on a Banach space $\mathcal{Z}$, a nonlinear map $F \in C(Z ; \mathcal{Z})$, where $Z$ is an open set in $\mathbb{R} \times \mathcal{Z}^{m}$.

Note that unique solvability issues for the Cauchy problem to multi-term linear equation of form (1) with Gerasimov-Caputo derivatives and bounded operators at them were studied in [8], various classes of nonlinear equations with Gerasimov-Caputo 
derivatives [9-11], or with a unique Riemann-Liouville derivative in a linear part of an equation $[12,13]$ have been studied before.

Linear equations of form (1) with Riemann-Liouville derivatives were studied in the work [14] in the case of bounded operators in the equation, and in [15] in the case of closed operators. In [14] it was shown, that the Cauchy type problem for an equation with several Riemann-Liouville derivatives has the so-called defect $m^{*}$, when several initial data must be zero in the lower order initial conditions for the solvability of the problem. So, a natural initial value problem for a multi-term equation of such type is, generally speaking, the incomplete Cauchy problem

$$
D_{t}^{\alpha-m+k} z\left(t_{0}\right)=z_{k}, k=m^{*}, m^{*}+1, \ldots, m-1 .
$$

Section 2 of this work contains the unique solvability theorem for linear $(F \equiv f(t))$ problem (1), (2) from the work [14].

In Section 3, firstly problem (1), (2) is reduced to the integro-differential equation

$$
z(t)=\sum_{p=m^{*}}^{m-1} Z_{p}\left(t-t_{0}\right) z_{p}+\int_{t_{0}}^{t} Z_{m-1}(t-s) F\left(s, D_{s}^{\alpha-m} z(s), \ldots, D_{s}^{\alpha-1} z(s)\right) d s,
$$

where $\left\{Z_{p}(t) \in \mathcal{L}(\mathcal{Z}): t>0\right\}, p=m^{*}, m^{*}+1, \ldots, m-1$ are the $p$-resolving families of operators for linear Equation (1). Next, under the condition of Lipschitzian continuity of the nonlinear operator $F$, using the theorem of contraction mapping for Equation (3), we prove the unique solvability of problem (1), (2) on a small enough interval.

Finally, in the last section a theorem of a local in time unique solution existence is obtained for initial-boundary value problems to a class of quasilinear equations with timefractional derivatives, where linear operators are polynomials of an elliptic self-adjoint operator, which is differential with respect to spatial variables.

\section{Preliminary Results}

Let us consider the fractional integral and fractional derivative of Riemann-Liouville with the initial point at $t_{0} \in \mathbb{R}$ :

$$
J_{t}^{\alpha} h(t):=\int_{t_{0}}^{t} \frac{(t-s)^{\alpha-1}}{\Gamma(\alpha)} h(s) d s, \quad D_{t}^{\alpha} h(t)=D_{t}^{m} J_{t}^{m-\alpha} h(t), \quad t>t_{0},
$$

where $m-1<\alpha \leq m \in \mathbb{N}$, i.e., $m:=\lceil\alpha\rceil$.

By $\mathfrak{L}[h]$ denote the Laplace transform of a function $h: \mathbb{R}_{+} \rightarrow \mathcal{Z}$. For the fractional integral and the fractional derivative of Riemann-Liouville we have the equalities [2]

$$
\mathfrak{L}\left[J_{t}^{\alpha} h\right](\lambda)=\lambda^{-\alpha} \mathfrak{L}[h](\lambda), \quad \mathfrak{L}\left[D_{t}^{\alpha} h\right](\lambda)=\lambda^{\alpha} \mathfrak{L}[h](\lambda)-\sum_{k=0}^{m-1} \lambda^{m-1-k} D_{t}^{\alpha-m+k} h(0),
$$

Hereafter $D_{t}^{\alpha-m+k} h(0):=\lim _{t \rightarrow 0+} D_{t}^{\alpha-m+k} h(t)$.

Let $\mathcal{Z}$ be a Banach space, $\mathcal{L}(\mathcal{Z})$ be the Banach space of bounded linear operators on $\mathcal{Z}$, $T>t_{0}$. Consider the inhomogeneous equation

$$
D_{t}^{\alpha} z(t)=\sum_{j=1}^{m-1} A_{j} D_{t}^{\alpha-m+j} z(t)+\sum_{l=1}^{n} B_{l} D_{t}^{\alpha_{l}} z(t)+\sum_{s=1}^{r} C_{s} J_{t}^{\beta_{s}} z(t)+f(t), t \in\left(t_{0}, T\right) .
$$

Here $0<\alpha_{1}<\alpha_{2}<\cdots<\alpha_{n}<\alpha, m_{l}:=\left\lceil\alpha_{l}\right\rceil, m:=\lceil\alpha\rceil, \alpha_{l}-m_{l} \neq \alpha-m$, $l=1,2, \ldots, n, \beta_{1}>\beta_{2}>\cdots>\beta_{r} \geq 0$, operators $A_{j}, j=1,2, \ldots, m-1, B_{l}, l=1,2, \ldots, n$, $C_{s}, s=1,2, \ldots, r$, are linear and bounded in $\mathcal{Z}$. Let 


$$
\begin{array}{ll}
\underline{\alpha}:=\max \left\{\alpha_{l}: l \in\{1,2, \ldots, n\}, \alpha_{l}-m_{l}<\alpha-m\right\}, & \underline{m}=\lceil\underline{\alpha}\rceil, \\
\bar{\alpha}:=\max \left\{\alpha_{l}: l \in\{1,2, \ldots, n\}, \alpha_{l}-m_{l}>\alpha-m\right\}, & \bar{m}=\lceil\bar{\alpha}\rceil .
\end{array}
$$

Denote by $m^{*}:=\max \{\underline{m}-1, \bar{m}\}$ the defect of the Cauchy type problem for Equation (4) [14].

A solution of the incomplete Cauchy type problem

$$
D_{t}^{\alpha-m+k} z\left(t_{0}\right)=z_{k}, k=m^{*}, m^{*}+1, \ldots, m-1,
$$

for (4) is a function $z:\left(t_{0}, T\right] \rightarrow \mathcal{Z}$ such that $J_{t}^{m-\alpha} z \in C^{m}\left(\left(t_{0}, T\right] ; \mathcal{Z}\right) \cap C^{m-1}\left(\left[t_{0}, T\right] ; \mathcal{Z}\right)$, $J_{t}^{m_{l}-\alpha_{l}} z \in C^{m_{l}}\left(\left(t_{0}, T\right] ; \mathcal{Z}\right), l=1,2, \ldots, n, J_{t}^{\beta_{s}} z \in C\left(\left(t_{0}, T\right] ; \mathcal{Z}\right), s=1,2, \ldots, r$, while equality (4) for $t \in\left(t_{0}, T\right]$ and (5) hold.

Put $\Gamma=\Gamma_{+} \cup \Gamma_{-} \cup \Gamma_{0}, \Gamma_{0}=\left\{\lambda \in \mathbb{C}:|\lambda|=r_{0}, \arg \lambda \in(-\pi, \pi)\right\}, \Gamma_{ \pm}=\{\lambda \in \mathbb{C}:$ $\left.\arg \lambda= \pm \pi,|\lambda| \in\left[r_{0}, \infty\right)\right\}$,

$$
\begin{gathered}
R_{\lambda}:=\left(I-\sum_{j=1}^{m-1} \lambda^{j-m} A_{j}-\sum_{l=1}^{n} \lambda^{\alpha_{l}-\alpha} B_{l}-\sum_{s=1}^{r} \lambda^{-\beta_{s}-\alpha} C_{s}\right)^{-1}, \\
Z_{p}(t)=\frac{1}{2 \pi i} \int_{\Gamma} \lambda^{-\alpha} R_{\lambda} \cdot\left(\lambda^{m-1-p} I-\sum_{j=p+1}^{m-1} \lambda^{j-1-p} A_{j}\right) e^{\lambda t} d \lambda, p=0,1, \ldots, m-1, t>0 .
\end{gathered}
$$

Substitute in ([14], Theorem 2) $t-t_{0}$ instead of $t$ and obtain the next result.

Theorem 1 ([14]). Let $m-1<\alpha \leq m \in \mathbb{N}, 0<\alpha_{1}<\alpha_{2}<\cdots<\alpha_{n}<\alpha, m_{l}-1<$ $\alpha_{l} \leq m_{l} \in \mathbb{N}, \alpha_{l}-m_{l} \neq \alpha-m, l=1,2, \ldots, n, \beta_{1}>\beta_{2}>\cdots>\beta_{r} \geq 0, A_{j} \in \mathcal{L}(\mathcal{Z})$, $j=1,2, \ldots, m-1, B_{l} \in \mathcal{L}(\mathcal{Z}), l=1,2, \ldots, n, C_{s} \in \mathcal{L}(\mathcal{Z}), s=1,2, \ldots, r, z_{k} \in \mathcal{Z}, k=$ $m^{*}, m^{*}+1, \ldots, m-1, f \in C\left(\left(t_{0}, T\right) ; \mathcal{Z}\right) \cap L_{1}\left(t_{0}, T ; \mathcal{Z}\right)$. Then there exists an unique solution to (4), (5). It has the form

$$
z(t)=\sum_{p=m^{*}}^{m-1} Z_{p}\left(t-t_{0}\right) z_{p}+\int_{t_{0}}^{t} Z_{m-1}(t-s) f(s) d s .
$$

\section{Quasilinear Equation}

Let $Z$ be an open set in $\mathbb{R} \times \mathcal{Z}^{m}, F: Z \rightarrow \mathcal{Z}$, consider the quasilinear equation

$$
\begin{aligned}
D_{t}^{\alpha} z(t) & =\sum_{j=1}^{m-1} A_{j} D_{t}^{\alpha-m+j} z(t)+\sum_{l=1}^{n} B_{l} D_{t}^{\alpha_{l}} z(t)+\sum_{s=1}^{r} C_{s} J_{t}^{\beta_{s}} z(t) \\
& +F\left(t, D_{t}^{\alpha-m} z(t), D_{t}^{\alpha-m+1} z(t), \ldots, D_{t}^{\alpha-1} z(t)\right) .
\end{aligned}
$$

A solution of the incomplete Cauchy type problem

$$
D_{t}^{\alpha-m+k} z\left(t_{0}\right)=z_{k}, k=m^{*}, m^{*}+1, \ldots, m-1,
$$

for Equation (6) on $\left(t_{0}, t_{1}\right]$ will be called such function $z \in C\left(\left(t_{0}, t_{1}\right] ; \mathcal{Z}\right)$, that $J_{t}^{m-\alpha} z \in$ $C^{m}\left(\left(t_{0}, t_{1}\right] ; \mathcal{Z}\right) \cap C^{m-1}\left(\left[t_{0}, t_{1}\right] ; \mathcal{Z}\right), J_{t}^{m_{l}-\alpha_{l}} z \in C^{m_{l}}\left(\left(t_{0}, t_{1}\right] ; \mathcal{Z}\right), l=1,2, \ldots, n$, and $J_{t}^{\beta_{s}} z \in$ $C\left(\left(t_{0}, t_{1}\right] ; \mathcal{Z}\right), s=1,2, \ldots, r$, the inclusion $\left(t, D_{t}^{\alpha-m} z(t), D_{t}^{\alpha-m+1} z(t), \ldots, D_{t}^{\alpha-1} z(t)\right) \in Z$ and equality (6) are valid for all $t \in\left(t_{0}, t_{1}\right]$, conditions (7) are fulfilled.

Let us introduce the notations $\bar{x}:=\left(x_{0}, x_{1}, \ldots, x_{m-1}\right) \in \mathcal{Z}^{m}, S_{\delta}(\bar{x})=\left\{\bar{y} \in \mathcal{Z}^{m-1}\right.$ : $\left.\left\|y_{k}-x_{k}\right\|_{\mathcal{Z}} \leq \delta, k=0,1, \ldots, m-1\right\}$. 
A mapping $F: Z \rightarrow \mathcal{Z}$ is called locally Lipschitzian in $\bar{x}$, if for every $(t, \bar{x}) \in Z$ there exist such $\delta>0, l>0$, that $[t-\delta, t+\delta] \times S_{\delta}(\bar{x}) \subset Z$, and for all $(s, \bar{y}),(s, \bar{v}) \in$ $[t-\delta, t+\delta] \times S_{\delta}(\bar{x})$ the inequality

$$
\|F(s, \bar{y})-F(s, \bar{v})\|_{\mathcal{Z}} \leq l \sum_{k=0}^{m-1}\left\|y_{k}-v_{k}\right\|_{\mathcal{Z}}
$$

is satisfied.

Lemma 1. Let $0<\alpha_{1}<\alpha_{2}<\cdots<\alpha_{n}<\alpha, m=\lceil\alpha\rceil, m_{l}=\left\lceil\alpha_{l}\right\rceil, \alpha_{l}-m_{l} \neq \alpha-m$, $l=1,2, \ldots, n, \beta_{1}>\beta_{2}>\cdots>\beta_{r} \geq 0, A_{j} \in \mathcal{L}(\mathcal{Z}), j=1,2, \ldots, m-1, B_{l} \in \mathcal{L}(\mathcal{Z})$, $l=1,2, \ldots, n, C_{s} \in \mathcal{L}(\mathcal{Z}), s=1,2, \ldots, r, z_{k} \in \mathcal{Z}, k=m^{*}, m^{*}+1, \ldots, m-1, Z$ be an open set in $\mathbb{R} \times \mathcal{Z}^{m},\left(t_{0}, 0,0, \ldots, 0, z_{m^{*}}, z_{m^{*}+1}, \ldots, z_{m-1}\right) \in Z, F \in C(Z ; \mathcal{Z})$. Then a function $z:\left(t_{0}, t_{1}\right] \rightarrow \mathcal{Z}$ is a solution of problem (6), (7) on $\left(t_{0}, t_{1}\right]$, if and only if $J_{t}^{m-\alpha} z \in C^{m-1}\left(\left[t_{0}, t_{1}\right] ; \mathcal{Z}\right)$ and for all $t \in\left(t_{0}, t_{1}\right]$

$$
z(t)=\sum_{p=m^{*}}^{m-1} Z_{p}\left(t-t_{0}\right) z_{p}+\int_{t_{0}}^{t} Z_{m-1}(t-s) F\left(s, D_{s}^{\alpha-m} z(s), \ldots, D_{s}^{\alpha-1} z(s)\right) d s
$$

Proof. If $z$ is a solution of problem (6), (7), then the mapping

$$
t \rightarrow F\left(t, D_{t}^{\alpha-m} z(t), D_{t}^{\alpha-m+1} z(t), \ldots, D_{t}^{\alpha-1} z(t)\right)
$$

acts continuously from $\left[t_{0}, t_{1}\right]$ into $\mathcal{Z}$ due to the definition of the solution at small enough $t_{1}-t_{0}$. By Theorem 2 (see [14]) a solution satisfies Equation (8).

Let $z$ satisfy Equation (8), then one can verify that $z$ is a solution to problem (6), (7) due to Theorem 1 [14] and by repeating word to word the proof of Lemma 3 in [14].

Theorem 2. Let $m-1<\alpha \leq m \in \mathbb{N}, 0<\alpha_{1}<\alpha_{2}<\cdots<\alpha_{n}<\alpha, m_{l}-1<\alpha_{l} \leq m_{l} \in \mathbb{N}$, $\alpha_{l}-m_{l} \neq \alpha-m, l=1,2, \ldots, n, \beta_{1}>\beta_{2}>\cdots>\beta_{r} \geq 0, A_{j} \in \mathcal{L}(\mathcal{Z}), j=1,2, \ldots, m-1$, $B_{l} \in \mathcal{L}(\mathcal{Z}), l=1,2, \ldots, n, C_{s} \in \mathcal{L}(\mathcal{Z}), s=1,2, \ldots, r, z_{k} \in \mathcal{Z}, k=m^{*}, m^{*}+1, \ldots, m-1, Z$ be an open set in $\mathbb{R} \times \mathcal{Z}^{m},\left(t_{0}, 0,0, \ldots, 0, z_{m^{*}}, z_{m^{*}+1}, \ldots, z_{m-1}\right) \in Z$, a mapping $F \in C(Z ; \mathcal{Z})$ is locally Lipschitzian in $\bar{x}$. Then there exists such $t_{1}>t_{0}$, that problem (6), (7) has an unique solution on $\left(t_{0}, t_{1}\right]$.

Proof. Take $y:=J_{t}^{m-\alpha} z \in C^{m-1}\left(\left[t_{0}, t_{1}\right], \mathcal{Z}\right)$, then $y^{(k)}=D_{t}^{\alpha-m+k} z, k=1,2, \ldots, m-1$. Then the mapping $t \rightarrow F\left(t, y(t), y^{(1)}(t), \ldots, y^{(m-1)}(t)\right)$ acts continuously from $\left[t_{0}, t_{1}\right]$ into $\mathcal{Z}$. By Lemma 1 it suffices to show that the equation

$$
y(t)=\sum_{p=m^{*}}^{m-1} J_{t}^{m-\alpha} Z_{p}\left(t-t_{0}\right) z_{p}+J_{t}^{m-\alpha} \int_{t_{0}}^{t} Z_{m-1}(t-s) F\left(s, y(s), y^{(1)}(s), \ldots, y^{(m-1)}(s)\right) d s
$$

has an unique solution $y \in C^{m-1}\left(\left[t_{0}, t_{1}\right], \mathcal{Z}\right)$ for some $t_{1}>t_{0}$.

It was proved in Theorem 1 [14] that $D_{t}^{\alpha-m+n} Z_{m-1}(0)=0, n=0,1, \ldots, m-2$. Since for all $p=0,1, \ldots, m-1$

$$
\left\|\frac{\lambda^{-\alpha} R_{\lambda}}{\mu-\lambda}\left(\lambda^{m-1-p} I-\sum_{j=p+1}^{m-1} \lambda^{j-1-p} A_{j}\right)\right\|_{\mathcal{L}(\mathcal{Z})} \leq \frac{C_{1}}{|\lambda|^{\alpha-m+2}},
$$

$\alpha-m+2>1$, so, $\mathfrak{L}\left[D_{t}^{\alpha-m+n} Z_{m-1}\right](\mu)=\mu^{n-m} R_{\mu}$, at $t \in\left[t_{0}, t_{1}\right], n=0,1, \ldots, m-2$, 


$$
\left\|D_{t}^{\alpha-m+n} Z_{m-1}(t)\right\|_{\mathcal{L}(\mathcal{Z})} \leq \frac{1}{2 \pi} \int_{\Gamma}\left\|\lambda^{n-m} R_{\lambda}\right\|_{\mathcal{L}(\mathcal{Z})}\left|e^{\lambda t}\right| d s \leq C_{2} \int_{\delta}^{\infty} r^{n-m} d r+C_{3} \leq C_{4} .
$$

At $n=m-1$ we have

$$
\begin{gathered}
D_{t}^{\alpha-1} Z_{m-1}(t)=\frac{1}{2 \pi i} \int_{\Gamma} \frac{R_{\lambda}}{\lambda} e^{\lambda t} d \lambda \\
=I+\frac{1}{2 \pi i} \int_{\Gamma} \lambda^{-1}\left(\sum_{j=1}^{m-1} \lambda^{j-m} A_{j}+\sum_{l=1}^{n} \lambda^{\alpha_{l}-\alpha} B_{l}+\sum_{s=1}^{r} \lambda^{-\beta_{s}-\alpha} C_{s}\right) R_{\lambda} e^{\lambda t} d \lambda,
\end{gathered}
$$

for $\lambda \in \Gamma$

$$
\left\|\lambda^{-1}\left(\sum_{j=1}^{m-1} \lambda^{j-m} A_{j}+\sum_{l=1}^{n} \lambda^{\alpha_{l}-\alpha} B_{l}+\sum_{s=1}^{r} \lambda^{-\beta_{s}-\alpha} C_{s}\right) R_{\lambda}\right\|_{\mathcal{L}(\mathcal{Z})} \leq \frac{C_{5}}{|\lambda|^{1+\delta}},
$$

where $\delta=\min \left\{1, \alpha-\alpha_{l}: l=1,2, \ldots, n\right\}$. Consequently, at $t \in\left[t_{0}, t_{1}\right]$

$$
\left\|D_{t}^{\alpha-1} Z_{m-1}(t)\right\|_{\mathcal{L}(\mathcal{Z})} \leq C_{6} .
$$

Let $\tau>0$ and $\delta>0$ be such that $\left[t_{0}, t_{0}+\tau\right] \times S_{\delta}(\bar{z}) \subset Z$, where $\bar{z}=(0,0, \ldots, 0$, $\left.z_{m^{*}}, z_{m^{*}+1}, \ldots, z_{m-1}\right)$ is constructed using initial data (7). Denote by $\mathcal{S}$ the set of functions $y \in C^{m-1}\left(\left[t_{0}, t_{0}+\tau\right] ; \mathcal{Z}\right)$ such that $\left\|y^{(q)}(t)\right\| \leq \delta, q=0,1, \ldots, m^{*}-1,\left\|y^{(k)}(t)-z_{k}\right\| \leq \delta$, $k=m^{*}, m^{*}+1, \ldots, m-1$ for $t_{0} \leq t \leq t_{0}+\tau$. We define a metric on $\mathcal{S}$

$$
d(y, v):=\sum_{k=0}^{m-1} \sup _{t \in\left[t_{0}, t_{0}+\tau\right]}\left\|y^{(k)}(t)-v^{(k)}(t)\right\|_{\mathcal{Z}}
$$

then $\mathcal{S}$ is a complete metric space.

Note that

$$
\begin{aligned}
& J_{t}^{m-\alpha} \int_{t_{0}}^{t} Z_{m-1}(t-s) F\left(s, y(s), y^{(1)}(s), \ldots, y^{(m-1)}(s)\right) d s \\
= & \int_{t_{0}}^{t} J_{t}^{m-\alpha} Z_{m-1}(t-s) F\left(s, y(s), y^{(1)}(s), \ldots, y^{(m-1)}(s)\right) d s .
\end{aligned}
$$

This equality can be proved by changing the order of integration in its left part.

Define for $y \in \mathcal{S}$

$G(y)(t):=\sum_{p=m^{*}}^{m-1} J_{t}^{m-\alpha} Z_{p}\left(t-t_{0}\right) z_{p}+\int_{t_{0}}^{t} J_{t}^{m-\alpha} Z_{m-1}(t-s) F\left(s, y(s), y^{(1)}(s), \ldots, y^{(m-1)}(s)\right) d s$

for $t \in\left[t_{0}, t_{0}+\tau\right]$. Let us prove that $G$ maps the metric space $\mathcal{S}$ into itself and it is a contraction operator, if $\tau>0$ is sufficiently small. Indeed, for $n=0,1, \ldots, m-1$

$$
\begin{gathered}
{[G(y)]^{(n)}(t)=\sum_{p=m^{*}}^{m-1} D_{t}^{\alpha-m+n} Z_{p}\left(t-t_{0}\right) z_{p}} \\
+\int_{t_{0}}^{t} D_{t}^{\alpha-m+n} Z_{m-1}(t-s) F\left(s, y(s), y^{(1)}(s), \ldots, y^{(m-1)}(s)\right) d s,
\end{gathered}
$$


since $D_{t}^{\alpha-m+n} Z_{m-1}(0)=0, n=0,1, \ldots, m-2$. By Theorem 2 [14] we have $G(y) \in$ $C^{m-1}\left(\left[t_{0}, t_{0}+\tau\right] ; \mathcal{Z}\right),[G(y)]^{(q)}\left(t_{0}\right)=0, q=0,1, \ldots, m^{*}-1,[G(y)]^{(k)}\left(t_{0}\right)=z_{k}, k=$ $m^{*}, m^{*}+1, \ldots, m-1$. Therefore, for $t \in\left[t_{0}, t_{0}+\tau\right]\left\|[G(y)]^{(q)}(t)\right\|_{\mathcal{Z}} \leq \delta, q=0,1, \ldots, m^{*}-1$, $\left\|[G(y)]^{(k)}(t)-z_{k}\right\|_{\mathcal{Z}} \leq \delta, k=m^{*}, m^{*}+1, \ldots, m-1$, for a small enough $\tau>0$. So, $G: \mathcal{S} \rightarrow \mathcal{S}$.

Denote $F(t, \bar{D} y(t)):=F\left(t, y(t), y^{(1)}(t), \ldots, y^{(m-1)}(t)\right)$ for brevity. We have at $n=$ $0,1, \ldots, m-1, t \in\left[t_{0}, t_{0}+\tau\right]$ due to (10), (11)

$$
\begin{gathered}
\left\|[G(y)]^{(n)}(t)-[G(v)]^{(n)}(t)\right\|_{\mathcal{Z}}=\left\|\int_{t_{0}}^{t} D_{t}^{\alpha-m+n} Z_{m-1}(t-s)(F(s, \bar{D} y(s))-F(s, \bar{D} v(s))) d s\right\| \\
\leq \tau \sup _{t \in\left[t_{0}, t_{0}+\tau\right]}\left\|D_{t}^{\alpha-m+n} Z_{m-1}(t)\right\|_{\mathcal{L}(\mathcal{Z})} l \sum_{k=0}^{m-1} \sup _{t \in\left[t_{0}, t_{0}+\tau\right]}\left\|y^{(k)}(t)-v^{(k)}(t)\right\|_{\mathcal{Z}} d s \\
\leq C_{7} \tau d(y, v) \leq \frac{d(y, v)}{2 m}
\end{gathered}
$$

for small enough $\tau$. Therefore, $d(G(y), G(v)) \leq \frac{1}{2} d(y, v)$, the operator $G$ has a unique fixed point $y_{0} \in \mathcal{S}$, it is an unique local solution of integro-differential Equation (9). Thus, there exists a unique solution to problem (6), (7) on the segment $\left[t_{0}, t_{0}+\tau\right]$, it is uniquely defined by the equality $z=D_{t}^{m-\alpha} y_{0}$.

\section{A Class of Initial-Boundary Value Problems}

Assume given the polynomials

$$
P_{1}(\lambda)=\sum_{p=0}^{v} a_{p} \lambda^{p}, P_{2}^{j}(\lambda)=\sum_{p=0}^{v} b_{p}^{j} \lambda^{p}, P_{3}^{l}(\lambda)=\sum_{p=0}^{v} c_{p}^{l} \lambda^{p}, P_{4}^{s}(\lambda)=\sum_{p=0}^{v} d_{p}^{s} \lambda^{p},
$$

$a_{p}, b_{p}^{j}, c_{p}^{l}, d_{p}^{s} \in \mathbb{C}, p=0,1, \ldots, v \in \mathbb{N}, j=1,2, \ldots, m-1, l=1,2, \ldots, n, s=1,2, \ldots, r$, $a_{v} \neq 0, \Omega \subset \mathbb{R}^{d}$ is a bounded domain with a smooth boundary $\partial \Omega$,

$$
\begin{aligned}
& (\mathcal{A} u)(\xi)=\sum_{|q| \leq 2 \rho} a_{q}(\xi) \frac{\partial|q|}{\mathcal{\zeta}(\xi)} \frac{\xi_{1}^{q_{1}} \partial \xi_{2}^{q_{2}} \ldots \partial \xi_{d}^{q_{d}}}{}, \quad a_{q} \in C^{\infty}(\bar{\Omega}),
\end{aligned}
$$

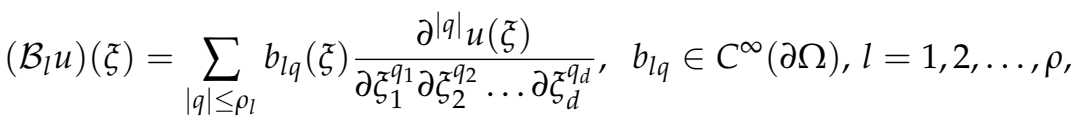

$q=\left(q_{1}, q_{2}, \ldots, q_{d}\right) \in \mathbb{N}_{0}^{d},|q|=q_{1}+\cdots+q_{d}$, and the operator pencil $\mathcal{A}, \mathcal{B}_{1}, \mathcal{B}_{2}, \ldots, \mathcal{B}_{\rho}$ is regularly elliptic [16]. Define the operator $\mathcal{A}_{1} \in \mathcal{C l}\left(L_{2}(\Omega)\right)$ with the domain

$$
D_{\mathcal{A}_{1}}=H_{\left\{\mathcal{B}_{l}\right\}}^{2 \rho}(\Omega):=\left\{v \in H^{2 \rho}(\Omega): \mathcal{B}_{l} v(\xi)=0, l=1,2, \ldots, \rho, \xi \in \partial \Omega\right\}
$$

by the rule $\mathcal{A}_{1} u:=\mathcal{A} u$. Suppose that $\mathcal{A}_{1}$ is a selfadjoint operator; then its spectrum $\sigma\left(\mathcal{A}_{1}\right)$ is real and discrete [16]. Moreover, assume that the spectrum $\sigma\left(\mathcal{A}_{1}\right)$ is bounded from the right and does not contain zero, $\left\{\varphi_{k}: k \in \mathbb{N}\right\}$ is an orthonormal in $L_{2}(\Omega)$ system of eigenfunctions of $\mathcal{A}_{1}$ in $L_{2}(\Omega)$ which is enumerated in nonincreasing order of the corresponding eigenvalues $\left\{\lambda_{k}: k \in \mathbb{N}\right\}$ with their multiplicities counted.

Take $m-1<\alpha \leq m \in \mathbb{N}, 0<\alpha_{1}<\alpha_{2}<\cdots<\alpha_{n}<\alpha, m_{l}-1<\alpha_{l} \leq m_{l} \in \mathbb{N}$, $\alpha_{l}-m_{l} \neq \alpha-m, l=1,2, \ldots, n, \beta_{1}>\beta_{2}>\cdots>\beta_{r} \geq 0, H: \mathbb{R}^{m+1} \rightarrow \mathbb{R}$. Denote by $m^{*}$ the defect of the Cauchy type problem, which is defined by the set of numbers $\alpha_{1}, \alpha_{2}, \ldots, \alpha_{n}, \alpha$ (see the second section), and consider the initial-boundary value problem

$$
D_{t}^{\alpha-m+k} u(\xi, 0)=u_{k}(\xi), k=m^{*}, m^{*}+1, \ldots, m-1, \quad \xi \in \Omega,
$$




$$
\begin{gathered}
\mathcal{B}_{l} \mathcal{A}^{k} u(\xi, t)=0, \quad k=0,1, \ldots, v-1, \quad l=1,2, \ldots, \rho, \quad(\xi, t) \in \partial \Omega \times\left(t_{0}, t_{1}\right], \\
P_{1}(\mathcal{A}) D_{t}^{\alpha} u(\xi, t)=\sum_{j=1}^{m-1} P_{2}^{j}(\mathcal{A}) D_{t}^{\alpha-m+j} u(\xi, t) \\
+\sum_{l=1}^{n} P_{3}^{l}(\mathcal{A}) D_{t}^{\alpha_{l}} u(\xi, t)+\sum_{s=1}^{r} P_{4}^{s}(\mathcal{A}) J_{t}^{\beta_{s}} u(\xi, t) \\
+H\left(\xi, D_{t}^{\alpha-m}(\xi, t), D_{t}^{\alpha-m+1}(\xi, t), \ldots, D_{t}^{\alpha-1}(\xi, t)\right), \quad(\xi, t) \in \Omega \times\left(t_{0}, t_{1}\right] .
\end{gathered}
$$

Put $\rho_{0} \geq 0, \mathcal{X}:=\left\{v \in H^{2 \rho v+\rho_{0}}(\Omega): \mathcal{B}_{l} \mathcal{A}^{k} v(\xi)=0, k=0,1, \ldots, v-1, l=\right.$ $1,2, \ldots, \rho, \xi \in \partial \Omega\}, \mathcal{Y}:=H^{\rho_{0}}(\Omega)$ is a Sobolev space $W_{2}^{\rho_{0}}(\Omega)$ for $\rho_{0}>0$, or the Lebesgue space $L^{\rho_{0}}(\Omega)$, if $\rho_{0}=0 ; L:=P_{1}(\mathcal{A}) \in \mathcal{L}(\mathcal{X} ; \mathcal{Y}), M_{j}:=P_{2}^{j}(\mathcal{A}) \in \mathcal{L}(\mathcal{X} ; \mathcal{Y}), j=1,2, \ldots, m-$ $1, N_{l}:=P_{3}^{l}(\mathcal{A}) \in \mathcal{L}(\mathcal{X} ; \mathcal{Y}), l=1,2, \ldots, n, S_{s}:=P_{4}^{s}(\mathcal{A}) \in \mathcal{L}(\mathcal{X} ; \mathcal{Y}), s=1,2, \ldots, r$.

If $P_{1}\left(\lambda_{k}\right) \neq 0$ for all $k \in \mathbb{N}$, then there exists the inverse operator $L^{-1} \in \mathcal{L}(\mathcal{Y} ; \mathcal{X})$ and (12)-(14) is representable in form (6), (7), where $\mathcal{Z}=\mathcal{X}, A_{j}=L^{-1} M_{j} \in \mathcal{L}(\mathcal{Z})$, $j=1,2, \ldots, m-1, B_{l}=L^{-1} N_{l} \in \mathcal{L}(\mathcal{Z}), l=1,2, \ldots, n, C_{s}=L^{-1} S_{s} \in \mathcal{L}(\mathcal{Z}), s=1,2, \ldots, r$, $z_{k}=u_{k}(\cdot), k=m^{*}, m^{*}+1, \ldots, m-1, F\left(x_{0}, x_{1}, \ldots, x_{m-1}\right)=L^{-1} H\left(\cdot, x_{0}, x_{1}, \ldots, x_{m-1}\right)$.

Theorem 3. Let $m-1<\alpha \leq m \in \mathbb{N}, 0<\alpha_{1}<\alpha_{2}<\cdots<\alpha_{n}<\alpha, m_{l}-1<\alpha_{l} \leq m_{l} \in \mathbb{N}$, $\alpha_{l}-m_{l} \neq \alpha-m, l=1,2, \ldots, n, \beta_{1}>\beta_{2}>\cdots>\beta_{r} \geq 0$, the spectrum $\sigma\left(\mathcal{A}_{1}\right)$ do not contain the origin and zeros of the polynomial $P_{1}, 4 \rho v+2 \rho_{0}>d, u_{k} \in \mathcal{X}, k=m^{*}, m^{*}+1, \ldots, m-1$, $H \in C^{\infty}\left(\Omega \times \mathbb{R}^{n} ; \mathbb{R}\right)$. Then at some $t_{1}>t_{0}$ there exists an unique solution of problem (12)-(14).

Proof. In this problem the domain of nonlinear operator is $Z=\mathbb{R} \times \mathcal{X}^{m}$ and due to the inequality $4 \rho v+2 \rho_{0}>d$ by Proposition 1 ([17], Appendix B) we have

$$
H\left(\cdot, x_{0}(\cdot), x_{1}(\cdot), \ldots, x_{n-1}(\cdot)\right) \in C^{\infty}\left(\mathcal{X}^{m} ; H^{2 \rho v+\rho_{0}}(\Omega)\right),
$$

hence, $F\left(x_{0}(\cdot), x_{1}(\cdot), \ldots, x_{m-1}(\cdot)\right):=L^{-1} H\left(\cdot, x_{0}(\cdot), x_{1}(\cdot), \ldots, x_{m-1}(\cdot)\right) \in C^{\infty}\left(\mathcal{X}^{m} ; \mathcal{X}\right)$. Then by Theorem 2 we obtain the statement of this theorem.

Example 1. Take $\alpha=5 / 2, m=3, n=1, r=1, \alpha_{1}=2 / 3, \beta_{1}=1 / 2, v=2, P_{1}(\lambda)=\lambda^{2}$, $P_{2}^{1}(\lambda)=b_{0}+b_{1} \lambda+b_{2} \lambda^{2}, P_{2}^{2}(\lambda) \equiv 0, P_{3}^{1}(\lambda)=c_{0}+c_{1} \lambda+c_{2} \lambda^{2}, P_{4}^{1}(\lambda)=d_{0}+d_{1} \lambda+d_{2} \lambda^{2}$, $d=1, \Omega=(0, \pi), \rho=1, \mathcal{A} u=\frac{\partial^{2} u}{\partial \xi^{2}}, \mathcal{B}_{1}=I$. Then $\underline{\alpha}:=\max \varnothing:=0, \underline{m}:=\lceil 0\rceil=0$, $\bar{\alpha}:=\max \{2 / 3\}=2 / 3, \bar{m}:=\lceil 2 / 3\rceil=1, m^{*}=1$, problem (12)-(14) has the form

$$
\begin{gathered}
D_{t}^{5 / 2} \frac{\partial^{4} u}{\partial \xi^{4}}(\xi, t)=\left(b_{0}+b_{1} \frac{\partial^{2}}{\partial \xi^{2}}+b_{2} \frac{\partial^{4}}{\partial \xi^{4}}\right) D_{t}^{1 / 2} u(\xi, t) \\
+\left(c_{0}+c_{1} \frac{\partial^{2}}{\partial \xi^{2}}+c_{2} \frac{\partial^{4}}{\partial \xi^{4}}\right) D_{t}^{2 / 3} u(\xi, t)+\left(d_{0}+d_{1} \frac{\partial^{2}}{\partial \xi^{2}}+d_{2} \frac{\partial^{4}}{\partial \xi^{4}}\right) J_{t}^{1 / 2} u(\xi, t) \\
+F\left(\xi, J_{t}^{1 / 2} u(\xi, t), D_{t}^{1 / 2} u(\xi, t), D_{t}^{3 / 2} u(\xi, t)\right), \quad(\xi, t) \in(0, \pi) \times\left(t_{0}, t_{1}\right], \\
u(0, t)=u(\pi, t)=\frac{\partial^{2} u}{\partial \xi^{2}}(0, t)=\frac{\partial^{2} u}{\partial \xi^{2}}(\pi, t)=0, \quad t \in\left(t_{0}, t_{1}\right], \\
D^{1 / 2} u(\xi, 0)=u_{1}(\xi), \quad D^{3 / 2} u(\xi, 0)=u_{2}(\xi) \quad \xi \in(0, \pi) .
\end{gathered}
$$

\section{Conclusions}

The local solvability is shown for the incomplete Cauchy type problem to a solved with respect to a highest derivative multi-term fractional differential equation with bounded operators at Riemann-Liouville derivatives in a Banach space with locally Lipschitzian nonlinear part. The results of the work [14] on inhomogeneous linear multi-term equation are used here for the research of the quasilinear equation, depending on lower order fractional derivatives with orders, which fractional part is equal to the fractional part of the highest fractional derivative. The abstract result was applied to the investigation of initial-boundary value problems to partial differential equations containing polynomials with respect to 
self-adjoint elliptic differential in spatial variables operator at time-fractional derivatives. Here the highest time-fractional partial derivative acts on the highest spatial derivative.

Our next step is to abandon this condition by allowing unlimited operators in an abstract equation. The linear case of this type has been investigated in [15], the nonlinear one has not yet been studied. Another significant step planned by the authors in the coming papers will be the rejection of conditions for the fractional part of the orders of derivatives on which the nonlinear operator depends (see above).

Author Contributions: Conceptualization, V.E.F. and B.T.K.; methodology, V.E.F. and B.T.K.; software, M.M.T.; validation, V.E.F. and M.M.T.; formal analysis, M.M.T.; investigation, M.M.T.; resources, V.E.F. and B.T.K.; data curation, M.M.T.; writing_original draft preparation, V.E.F. and M.M.T.; writing - review and editing, V.E.F. and B.T.K.; visualization, M.M.T.; supervision, V.E.F.; project administration, V.E.F.; funding acquisition, V.E.F. All authors have read and agreed to the published version of the manuscript.

Funding: The work is supported by the Russian Foundation for Basic Research and Vietnam Academy of Science and Technology, grants numbers 21-51-54003 and QTRU 01-01/21-22, the first two coauthors are funded also by the grant of President of the Russian Federation to support leading scientific schools, project number NSh-2708.2022.1.1.

Data Availability Statement: Not applicable.

Conflicts of Interest: The authors declare no conflict of interest. The funders had no role in the design of the study; in the collection, analyses, or interpretation of data; in the writing of the manuscript, or in the decision to publish the results.

\section{References}

1. Samko, S.G.; Kilbas, A.A.; Marichev, O.I. Fractional Integrals and Derivatives. Theory and Applications; Gordon and Breach Science Publishers: Philadelphia, PA, USA, 1993.

2. Podlubny, I. Fractional Differential Equations; Academic Press: Boston, MA, USA, 1999.

3. Nakhushev, A.M. Fractional Calculus and Its Applications; Fizmatlit: Moscow, Russia, 2003. (In Russian)

4. Kilbas, A.A.; Srivastava, H.M.; Trujillo, J.J. Theory and Applications of Fractional Differential Equations; Elsevier Science Publishing: Amsterdam, The Netherlands; Boston, MA, USA; Heidelberg, Germany, 2006.

5. Kostić, M. Abstract Volterra Integro-Differential Equations; CRC Press: Boca Raton, FL, USA, 2015.

6. Tarasov, V.E. Fractional Dynamics: Applications of Fractional Calculus to Dynamics of Particles, Fields and Media; Springer: New York, NY, USA, 2011.

7. Uchaykin, V.V. Fractional Derivatives for Physicists and Engineers; Higher Education Press: Beijing, China, 2012.

8. Fedorov, V.E.; Boyko, K.V.; Phuong, T.D. Initial value problems for some classes of linear evolution equations with several fractional derivatives. Math. Notes NEFU 2021, 28, 85-104.

9. Plekhanova, M.V. Nonlinear equations with degenerate operator at fractional Caputo derivative. Math. Methods Appl. Sci. 2016, 40, 41-44. [CrossRef]

10. Plekhanova, M.V.; Baybulatova, G.D. Semilinear equations in Banach spaces with lower fractional derivatives. In International Conference in Nonlinear Analysis and Boundary Value Problems; Springer: Cham, Switzerland, 2019; Volume 292, pp. 81-93.

11. Fedorov, V.E.; Phuong, T.D.; Kien, B.T.; Boyko, K.V.; Izhberdeeva, E.M. A class of semilinear distributed order equations in Banach spaces. Chelyabinsk Phys. Math. J. 2020, 5, 343-351.

12. Fedorov, V.E.; Avilovich, A.S. Semilinear fractional-order evolution equations of Sobolev type in the sectorial. Complex Var. Elliptic Equ. 2021, 66, 1108-1121. [CrossRef]

13. Fedorov, V.E.; Nagumanova, A.V.; Avilovich, A.S. A class of inverse problems for evolution equations with the Riemann-Liouville derivative in the sectorial case. Math. Methods Appl. Sci. 2021, 44, 11961-11969. [CrossRef]

14. Fedorov, V.E.; Turov, M.M. The defect of a Cauchy type problem for linear equations with several Riemann-Liouville derivatives. Sib. Math. J. 2021, 62, 925-942. [CrossRef]

15. Fedorov, V.E.; Du, W.-S.; Turov, M.M. On the unique solvability of incomplete Cauchy type problems for a class of multi-term equations with the Riemann-Liouville derivatives. Symmetry 2022, 14, 75. [CrossRef]

16. Triebel, H. Interpolation Theory. Function Spaces. Differential Operators; Deutscher Verlag des Wissenschaften: Berlin, Germany, 1978.

17. Hassard, B.D.; Kazarinoff, N.D.; Wan, Y.-H. Theory and Applications of Hopf Bifurcation; Cambridge University Press: Cambridge, UK, 1981. 